\title{
BMJ Open Evaluating the impact of a community- based social prescribing intervention on people with type 2 diabetes in North East England: mixed-methods study protocol
}

\author{
Suzanne Moffatt, ${ }^{1}$ John Wildman, ${ }^{2}$ Tessa M Pollard, ${ }^{3}$ Linda Penn, ${ }^{1}$ Nicola O'Brien, ${ }^{1}$ \\ Mark S Pearce, ${ }^{1}$ Josephine M Wildman ${ }^{1}$
}

To cite: Moffatt S, Wildman J, Pollard TM, et al. Evaluating the impact of a communitybased social prescribing intervention on people with type 2 diabetes in North East England: mixed-methods study protocol. BMJ Open 2018;9:e026826. doi:10.1136/ bmjopen-2018-026826

- Prepublication history for this paper is available online. To view these files, please visit the journal online (http://dx.doi. org/10.1136/bmjopen-2018026826).

Received 26 September 2018 Revised 5 November 2018 Accepted 7 November 2018

Check for updates

(C) Author(s) (or their employer(s)) 2018. Re-use permitted under CC BY-NC. No commercial re-use. See rights and permissions. Published by BMJ.

${ }^{1}$ Institute of Health and Society, Newcastle University, Newcastle upon Tyne, UK

${ }^{2}$ Business School, Newcastle University, Newcastle upon

Tyne, UK

${ }^{3}$ Department of Anthropology, Durham University, Durham, UK

Correspondence to

Dr Suzanne Moffatt;

suzanne.moffatt@ncl.ac.uk

\section{ABSTRACT}

Introduction Social prescribing enables healthcare professionals to use voluntary and community sector resources to improve support for people with longterm conditions. It is widely promoted in the UK as a way to address complex health, psychological and social issues presented in primary care, yet there is insufficient evidence of effectiveness or value for money. This study aims to evaluate the impact and costs of a link-worker social prescribing intervention on the health and healthcare use of adults aged 40-74 with type 2 diabetes, living in a multi-ethnic area of high socioeconomic deprivation.

Methods and analysis Mixed-methods approach combining (1) quantitative quasi-experimental methods to evaluate the effects of social prescribing on health and healthcare use and cost-effectiveness analysis and (2) qualitative ethnographic methods to observe how patients engage with social prescribing. Quantitative data comprise Secondary Uses Service data and Quality Outcomes Framework data. The primary outcome is glycated haemoglobin, and secondary outcomes are secondary care use, systolic blood pressure, weight/ body mass index, cholesterol and smoking status; these data will be analysed longitudinally over 3 years using four different control conditions to estimate a range of treatment effects. The ranges where the intervention is cost-effective will be identified from the perspective of the healthcare provider. Qualitative data comprise participant observation and interviews with purposively sampled service users, and focus groups with link-workers (intervention providers). Analysis will involve identification of themes and synthesising and theorising the data. Finally, a coding matrix will identify convergence and divergence among all study components.

Ethics and dissemination UK NHS Integrated Research Approval System Ethics approved the quantitative research (Reference no. 18/L0/0631). Durham University Research Ethics Committee approved the qualitative research. The authors will publish the findings in peer-reviewed journals and disseminate to practitioners, service users and commissioners via a number of channels including professional and patient
Strengths and limitations of this study

- This study addresses the current evidence gap regarding the impact and value for money of a link-worker social prescribing intervention.

- This is the first study to combine natural experimental methods, economic assessment and ethnography to measure the impact of social prescribing.

- This study is timely and relevant to patients with type 2 diabetes, the NHS and the voluntary and community sectors.

- The focus on type 2 diabetes enables selection of relevant outcome measures, but limits generalisability to other long-term conditions.

- Further challenges in generalising the findings arise because social prescribing models vary in terms of commissioning, funding, referral criteria and pathway, service provider and the range of 'prescribed' activities.

networks, conferences and social media. Results will be disseminated via peer-reviewed journals.

\section{BACKGROUND}

Social prescribing enables healthcare professionals to address non-medical causes of ill-health through using the resources of the voluntary and community sectors. ${ }^{1}$ Social prescribing is widely promoted in the UK as a way of addressing complex health, psychological and social issues presented in primary care, ${ }^{2}$ improving support for people with longterm conditions and making general practice more sustainable. ${ }^{3}$ It is also viewed as an intervention with the potential to reduce health inequalities. $^{23}$ There is no agreed definition and no single model of social prescribing. However, typically social prescribing for people with long-term conditions harnesses voluntary and community sector assets 
to encourage self-care and facilitate health-creating communities. ${ }^{2-4}$ To encourage engagement, most social prescribing schemes involve a facilitator 'link-worker' who supports service users to identify and achieve personalised condition management and behaviour change goals. $^{5}$

There is considerable support at policy level in the UK for social prescribing ${ }^{67}$ and the UK's Department of Health recently pledged $£ 4.5$ million towards social prescribing in primary care. ${ }^{8}$ However, a systematic review of the effectiveness of social prescribing interventions ${ }^{9}$ concluded that there is currently insufficient robust evidence of effectiveness or value for money. This review reinforces the conclusions of three non-systematic reviews advocating that evaluations of community-based social prescribing require control groups, larger sample sizes, longer-term follow-up and clinically meaningful outcome measures. ${ }^{41011}$

Of the two most robust studies available, the first published in $2000^{12}$ (included in Bickerdike $e$ t al's systematic review) was conducted in England and comprised a randomised controlled trial (RCT) of a link-worker intervention for primary care patients (aged 16 years and over) with psychosocial problems. Four months after randomisation, significant improvements were found for anxiety and some aspects of quality of life, but no effects were found for depression, social isolation, general practitioner (GP) consultation rate or GP prescribing in a sample of 161 patients ( $n=90$ in the intervention group; $n=71$ in the control group). The second study ${ }^{13}$ comprised a quasi-experimental general-practice level cluster RCT with a mixed-methods process evaluation of a practice-based Community Links Practitioner social prescribing intervention in 15 general practices in Scotland targeting patients with complex needs. ${ }^{13}$ This RCT did not find statistically significant differences in the primary outcome measureEQ-5D-5L-at 9 months compared with usual care, but improvements were reported in the following secondary outcomes: increased levels of self-reported exercise, reduced anxiety and depressive symptoms. There were no beneficial effects on self-reported healthcare use. The authors conclude that a longer-term outcome evaluation using robust routinely collected data is required. ${ }^{14}$

There is therefore an imperative to obtain robust evidence about the impact and cost-effectiveness of social prescribing. ${ }^{9}$ Building on recently completed quantitative and qualitative research, ${ }^{15-17}$ this study aims to evaluate the impact and costs of a community-based linkworker social prescribing intervention on the health and healthcare use of adults aged 40-74 with type 2 diabetes (T2D), living in a multi-ethnic area of high socioeconomic deprivation. Diabetes is a major public health issue; the number of people in the UK with this condition is expected to rise to over 6 million by $2035,85-90 \%$ of whom have T2D. If no changes are made to the treatment of T2D, the costs to the NHS are estimated to increase to $£ 17$ bn by 2035 , with associated increases in the wider costs to society estimated at over £22bn. ${ }^{18}{ }^{19}$ People with
T2D often have one or more other long-term condition, ${ }^{20}$ and T2D is often associated with mental health conditions, such as anxiety and depression, which can negatively affect an individual's ability to manage their T2D and other long-term conditions. ${ }^{21}$

The study takes a mixed-methods approach combining quantitative quasi-experimental methods to evaluate the effects of social prescribing on health and healthcare use and qualitative ethnographic methods to observe how patients engage with social prescribing and the range of its impacts on patients and their wider social networks. A cost-effectiveness analysis will also be undertaken.

This study aims to answer the following questions:

1. Does a link-worker social prescribing intervention targeting adults aged 40-74 with T2D result in changes to glycated haemoglobin (HbAlc), body mass index (BMI), systolic blood pressure, cholesterol, smoking, EQ-5D-5L and healthcare use from baseline to 12 months?

2. Does the intervention demonstrate greater effectiveness in subgroups (gender, age, and ethnicity) of the eligible population?

3. Does the intervention lead to improved health-related quality of life (measured by EQ-5D-5L) at 12 months?

4. How cost-effective is a link-worker social prescribing intervention targeting adults aged $40-74$ with T2D?

5. How does link-worker social prescribing lead to changes in the daily lives of individuals, their families and wider social networks?

6. To what extent does link-worker social prescribing reduce health inequalities?

\section{Intervention and study setting}

'Ways to Wellness' (http://waystowellness.org.uk/) delivers link-worker social prescribing to people aged $40-74$ with at least one of eight long-term conditions. It is based on extensive pilot work and the social prescribing model comprises community-based link-workers who deliver the intervention. Box 1 summarises the range and scope of the intervention.

The setting is a multi-ethnic inner-city area of high socioeconomic deprivation (population $\mathrm{n}=111557$ ), ranked 40th most deprived in England according to the Index of Multiple Deprivation. ${ }^{22}$ In the locality covered by the intervention, all-cause standardised mortality rates ( $<75$ years) are 150 , and emergency hospital admissions rates are 134, demonstrating considerable health disadvantage compared with the English standard of $100 .{ }^{23}$ Twenty-three per cent of the population in the intervention locality are from Black and minority ethnic communities compared with the Newcastle city and English averages of $15 \%{ }^{24}$

\section{Study population}

Community-dwelling adults aged 40 to 74 years, with T2D with or without comorbidity or disease-related complications or a diagnosis of depression or anxiety. The intervention group comprises people with T2D who meet with 


\section{Box 1 Ways to Wellness intervention}

- Ways to Wellness (http://waystowellness.org.uk/) is a community-based link-worker social prescribing intervention for people with long-term conditions. Intervention development was based on extensive pilot work and co-produced with people with long-term conditions, over 8 years (2007 to 2015). ${ }^{41-43}$ Ways to Wellness is managed via Ways to Wellness Ltd, a special-purpose vehicle set up to oversee the delivery of the service.

- The service began in April 2015 and is on target to recruit approximately 1500 participants annually over 7 years (total $n=10500$ ). Approximately $41 \%$ of these people have type 2 diabetes.

- Ways to Wellness aims to improve health-related outcomes and quality of life of people with long-term conditions by increasing their confidence and ability to manage their illness, and to reduce costs and/or improve value to the NHS in their treatment. The Ways to Wellness intervention has four key objectives: (1) to improve participants' health-related behaviours, (2) to improve self-care, (3) to encourage better long-term condition self-management and (4) to promote social integration. The strong focus on addressing the wider determinants of health through link-worker facilitated access to services like welfare rights, debt and housing advice and employment support emphasises the potential for the intervention to address health inequalities.

- Ways to Wellness is designed to provide an efficient referral mechanism for primary care practitioners, ${ }^{5}$ optimising referral practices through the facilitation of patients' access to community-based support and social engagement, thus supplementing the care patients receive from the NHS. ${ }^{44} 45$ The Ways to Wellness intervention is delivered by not-for-profit organisations (originally four, now two organisations) allocated to geographical clusters of GP practices. Patients are referred by their GP to Ways to Wellness and assigned to a link-worker, who is a trained facilitator. Link-workers use behaviour change techniques including motivational interviewing, goal-setting and feedback to promote the aims of Ways to Wellness. Goal identification and setting and progress monitoring is conducted using the 'Well-being Star' ${ }^{46}$ self-assessment tool. This proprietary tool helps service users to assess their state across eight parameters (lifestyle; self-care; symptom management; work, volunteering and activity; money; home environment; personal relationships; positive feeling).

- Link-workers provide the following for each of their assigned clients: (a) individual assessment, motivational interviewing and action planning ${ }^{47}$; (b) completion of the 'Well-being Star' self-assessment tool at baseline and 6 monthly up to 24 months; (c) support and guidance to access community services (eg, walking groups, physical activity classes and welfare rights advice); (d) promotion of volunteering opportunities; (e) promotion of improved self-care. The Ways to Wellness intervention is highly personalised involving face-to-face contacts in community settings and, where necessary, domiciliary visits. Contact is also made, where appropriate, via email, text and telephone. All contacts vary in duration and frequency in accordance with individual client need, but overall engagement with Ways to Wellness can be up to 2 years.

their link-worker at least once and complete a baseline assessment between April 2015 and June 2018.

\section{Study design}

Three separate work packages (WPs) will be undertaken, concluding with a fourth WP combining and integrating the different datasets.

\section{Work package 1}

Exploits the geographical implementation of the intervention as a natural experiment. Primary and secondary outcomes of individuals who engage with the intervention will be compared with those who are eligible but do not take part over 3 years from the intervention start date. Robustness of estimated effects will be investigated by exploiting a range of possible control groups (see table 1) and a falsification test.

\section{Outcome measures}

The primary outcome measure is HbAlc, a marker of blood glucose concentration. HbAlc is selected because it provides a good measure of T2D control, it is the diabetes management measure used by clinicians and is routinely collected in primary care. ${ }^{25}$ Secondary outcome measures are systolic blood pressure, weight/BMI, cholesterol and smoking status (all also routinely collected in primary care), quality of life and secondary care use.

Quality-of-life data (measured by EQ-5D-5L) are not routinely collected and are being collected by linkworkers. These data will provide a within-cohort comparison for $\mathrm{n}=1100$ individuals recruited between July 2018 and June 2019.

\section{Recruitment and sample size}

The numbers of people with T2D who have been referred to the intervention are as follows: April 2015-March 2016, $\mathrm{n}=462$; April 2016-March 2017, n=540; April 2017-March 2018, $\mathrm{n}=600$. We expect similar numbers of individuals for our control groups (ie, those with T2D not referred). With group sizes of over 1600, assuming type 1 error of $5 \%$ and one observation per participant, we have over $85 \%$ power to detect an effect size (standardised difference) of 0.15 in a continuous outcome and approximately $85 \%$ power to detect a difference of 5 percentage points in a binary outcome. We will cluster SEs from multiple regressions on individuals and at the primary care practice level to acknowledge that observations are not independent draws from the population.

The EQ-5D-5L data will be collected for all individuals referred to the intervention between July 2018 and June 2019. This gives a projected sample size of 1100 individuals meaning that power will be reduced in this group. To detect an effect size (standardised difference) of 0.2 , we would have a power of approximately $70 \%$.

\section{Research methods}

Using Secondary Uses Service data and Quality Outcomes Framework data, linked by North of England Commissioning Service, we will follow individual-level data over time from before the intervention period, across the intervention, and then for the length of the study in order to analyse the average treatment effect (which we call $\tau$ ) of the intervention. We will apply longitudinal data analysis, taking account of individual heterogeneity, as well as GP, time and provider fixed effects in order to control for unobservables and time-invariant selection effects 


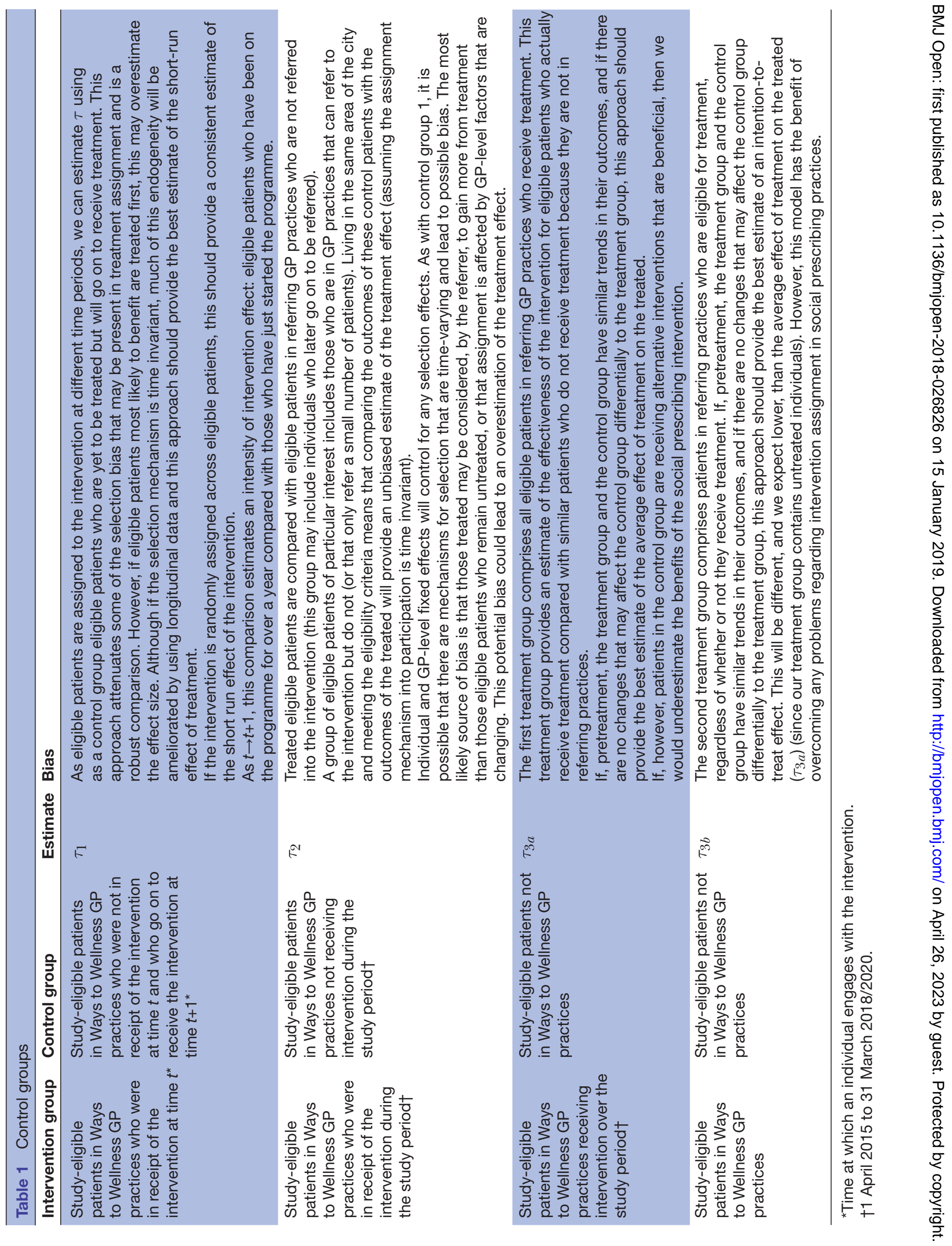


that may introduce bias into our estimates. Potential confounding variables will be included as extra controls where necessary. With observational data, it is often not possible to produce perfectly unbiased treatment effects. ${ }^{26}$ The best alternative is to provide a range of estimated treatment effect values, with a clear explanation of the underlying samples from which they are derived. Providing bounds for the treatment effects is more informative than restricting the analysis to a single control group in the expectation that all of the necessary assumptions hold. We will estimate the appropriate models for outcomes (depending on whether they are continuous or ordinal) using difference-in-differences techniques. These methods will be applied to a number of control groups identified in table 1 to provide estimates of the treatment effect: $\tau_{O L S}$ (from a naive estimation); $\tau_{1}$, exploiting the timing of the intervention to provide a control group; and $\tau_{2}$, exploiting the treatment assignment rules from within the social prescribing providing practices to identify a control group. Further, using routinely collected data from GP records in the North of England Commissioning Service database allows us to broaden our consideration of the control group. Within the Newcastle uponTyne GP practices covered by North of England Commissioning Service, only 17 out of 64 are able to refer to the social prescribing pathway. The availability of social prescribing is, therefore, an exogenous event, whereby individuals are unable to select whether they are in a social prescribing referring practice (it is possible that patients could switch from a non-social prescribing GP practice to a referring practice; however, we would consider such changes to be unlikely or small in number). By selecting GP practices that do not refer patients to social prescribing within Newcastle upon Tyne, we will use the treatment assignment rules (patients with T2D aged between 40 and 74) to generate a control group of patients from GPs outside the social prescribing referral network who would be eligible for treatment had social prescribing been available. Comparable control GP practices will be selected on the basis of practice Index of Multiple Deprivation scores to the social prescribing referral practices. In this case, the control group is exogenously determined: the individuals cannot select into treatment. This approach generates two possible control groups, $\tau_{3 a}$ and $\tau_{3 b}$, both of which will provide a different estimate of the treatment effect. With the larger samples sizes provided by control groups 2 and 3, we will be able to consider subgroup analyses (age, gender, ethnicity, presence of multimorbidity, service provider) to investigate whether there are some individuals who benefit more from treatment than others. Heterogeneity among treated groups is of serious concern to health policy-makers, and it may be that social prescribing has greater impact at different ages or among individuals with more comorbidity.

Our estimation strategy allows the estimation of a number of different average treatment effects that may or may not be affected by different levels of bias. We would expect:

$$
\tau_{O L S}>\tau_{1} \geq \tau_{2} \geq \tau_{3 a}>\tau_{3 b}
$$

For continuous outcomes, naïve ordinary least squares estimation would give an estimated treatment effect of $\hat{T}_{O L S}$. However, we would expect this estimate to overestimate any effect of treatment because individuals who are treated may be systematically different to patients who are untreated; for example, individuals who are untreated may have fewer comorbidities than the treated. Our intention-to-treat analysis $\left(\tau_{3 b}\right)$ should provide the smallest estimate of the treatment effect. Other estimates from the other control groups should lie between these upper and lower bounds. By highlighting the strengths and weaknesses of each estimated effect, our approach provides policy-makers with a much clearer understanding of the potential benefits that may arise from link-worker social prescribing. As a robustness test, we will undertake a falsification test where we will estimate the models outlined above using a pretreatment time period as the intervention date. In this case, any estimated treatment effect should be zero. We will estimate models on complete cases and also weighted samples in order to account for attrition.

\section{Work package 2}

Cost-effectiveness analysis ${ }^{27}$ will be undertaken from the perspective of the healthcare provider. Separate analyses will be conducted on primary and secondary outcome measures. Detailed costs of the programme are available. Outcomes are measured 1 and 3 years after follow-up, so appropriate discounting will be applied. The comparator will be the standard treatment regime of the non-intervention group. Effectiveness measures will come from WP1. Robustness will be investigated using sensitivity analysis. Outcome measures are incremental cost-effectiveness ratios demonstrating the ratio of the differences in the costs between the intervention and comparator and the difference in benefits.

\section{Research methods}

The intervention will be compared with the standard treatment regime of the non-intervention group, and costs and benefits, where appropriate, will be discounted at $1.5 \%$ in line with the guidelines from the National institute for Health and Care Excellence for public health interventions. ${ }^{28}$ The effectiveness data will use the estimates from WP1 including EQ-5D-5L, which will be expanded by extrapolating broader health outcomes based on the literature linking T2D to quality-adjusted life years. ${ }^{29}$ The range of estimated effects from WP1 will be applied in order to produce a range of cost-effectiveness ratios that will demonstrate, using incremental cost-effectiveness ratios, the ranges where the intervention is cost-effective (and potentially those ranges where it is not cost-effective).

Sensitivity of the results to changes in benefits and costs will be investigated by using the results from WP1. WP1 will provide a range of control groups that will provide different comparator groups, and also a range 
of estimated treatment effects. The sensitivity of the economic evaluation to these different estimates will be investigated as part of the economic evaluation.

The incremental cost-effectiveness ratio will provide a range of estimates that can be used to inform decision-makers. The role of (economic) evaluation is not to provide an answer as to whether an intervention should be adopted or not-it is to provide information to the decision-maker so they can decide based on all of the available evidence. ${ }^{30}$

\section{Work package 3}

Over an 18-month period, interviews, participant observation and focus groups will yield a detailed account of the experiences of participants. Data will be used to build an understanding of how and why the intervention works and for whom, as well as reasons why the intervention does not work and for whom.

Recruitment and sample size. Between 18 and 24 key participants (service users) will be recruited. Key participants will be purposively sampled on the basis of age, gender, ethnicity, employment status, social class, service provider, length of time engaged with the intervention and reason for remaining with/leaving the intervention. We plan to recruit friends and family opportunistically during participant observation to provide supplementary data and we will undertake semistructured interviews with around 12 family members and friends to explore their perspectives on the intervention. We will conduct focus groups with up to 22 link-workers.

\section{Research methods}

Each key participant will be followed from the beginning to the end of fieldwork to allow us to follow their 'social prescribing journey', including those who remain engaged with the service, those who 'complete' and those who drop out. We will observe their engagement with services, and explore practices within their families and social networks. At the start of fieldwork, key participants will participate in a semistructured interview exploring their experiences of the intervention. Each participant will be interviewed again in the final 6 months of fieldwork to follow up their experiences of the intervention. Between the two interviews, opportunities for participant observation will be pursued as far as is feasible and will include spending time in participants' homes and accompanying them to appointments with their link-worker and prescribed activities (eg, walking groups, volunteering activities, etc) depending on the preferences of the participant. Further episodes of participant observation will be pursued opportunistically. With at least 12 participants, particularly those who are less willing to invite the researcher into their lives for participant observation, we will use photo-elicitation interviews to supplement the standard interviews and provide a more complete and complex picture of the intervention. ${ }^{31} 32$ All link-workers will be invited to participate in focus groups. Further information on the delivery of Ways to Wellness will be obtained by joining intervention participants when they visit link-workers (where participants agree to this), which will include participant observation ('hanging out') in waiting rooms and staff rooms to build a picture of the 'culture' of each provider.

Detailed field notes will be recorded after each episode of participant observation, in line with standard ethnographic procedures. All interviews will be digitally recorded and subsequently transcribed verbatim. Qualitative data will comprise semistructured interview transcripts and ethnographic field notes recording meetings with link-workers and other episodes of participant observation. Detailed field notes will record scenes encountered, pursuing meanings and reflecting on positionality, and will supplement interviews by describing contexts and information that is not audio-recorded, such as non-verbal communication. Line-by-line coding will be conducted to identify emerging themes. Analysis will involve synthesising and theorising the data, and considering it in the context of other research. ${ }^{33}$ Participants' photographs will also be analysed in their own right, following Pink's suggestion that they can help the ethnographer to understand how participants represent themselves, their environments and experiences. ${ }^{34}$

\section{Work package 4}

Work package 4 integrates the findings of work packages 1,2 and 3. This is to enable a full interrogation of the compiled data for consistencies and differences, ensuring that the strengths of the complementary methods are fully integrated. Findings from each work package will be extracted and entered into a 'convergence coding matrix' ${ }^{35}$ a mixed-methods analysis technique that enables identification of where there is agreement, partial agreement or dissonance between the findings of different study components. ${ }^{35}$ This facilitates an analysis of the reasons for agreement or differences between the various datasets, enabling the complementary strengths of the quantitative and qualitative methods to be fully examined.

\section{Patient and public involvement}

Research questions and outcome measures were informed by $n=30$ patients referred to Ways to Wellness via a qualitative interview study ${ }^{16}$ which explored their priorities, experiences and preferences. Link-workers delivering the intervention informed the study via a set of focus groups and interviews in which they discussed operationalising the intervention.

Results will be disseminated to participants via Ways to Wellness, link-workers, the study website and through social media. For participants in the qualitative study, those findings will be disseminated via face-to-face meetings, printed literature and the study website.

\section{Summary}

This study is the first of its kind to take a mixed-methods approach that combines quantitative quasi-experimental 
methods, economic assessment and ethnography to assess the impact and value for money of a social prescribing intervention. The study is timely and relevant to the NHS and the voluntary and community sectors. Three elements make it distinctive.

First is the use of quasi-experimental methods. The social prescribing intervention can be considered as a natural experiment; that is, assignment to treatment is non-random by means of administrative selection. ${ }^{36} 37$ Although natural experimental methods are increasingly being used to evaluate public health interventions, ${ }^{37}$ applying these methods to measuring the impact of social prescribing is unique. Natural experimental methods divide the population into a treated and an untreated group, allowing the application of regression methods to estimate treatment effects. These methods have an advantage over randomised controlled design methods in that they can be applied to the evaluation of interventions that have already started, where the intervention is not under the control of the researcher and blinding assumptions that would usually be required for conducting a robust RCT would be violated.

A second distinctive aspect of this study is the use of ethnography. While standard interview approaches, as used earlier, ${ }^{16}$ are informative and valuable, ethnography is a more powerful tool to uncover how the intervention plays out in the complex daily lives and practices of participants. The use of observation, interviews, focus groups and photo-elicitation techniques provides contrasts between the 'life that is told' (accessed via interviews) and the 'life that is lived' (accessed via participant observation).$^{38}$ Ethnography enables examination of how the intervention fits in with the constraints of comorbidity, domestic routines and habits, (un)employment and the welfare system, and how the meanings of practices such as eating, exercising or taking part in community activities influence participants' engagement. Wider implementation will be enhanced by an understanding of how change is brought about, experienced and maintained. ${ }^{39}$ We will also examine inevitable variation in the delivery of the intervention (within the constraints of the protocol) by exploring any differences in the experiences of participants assigned to the two different provider organisations.

Third, we combine natural experimental methods and ethnography in a distinctive mixed-methods approach. These work packages will run in parallel, with the findings from one approach informing the other, and vice versa. The longitudinal nature of the quantitative data enables us to quantify impacts between different groups over time; the ethnography enables an approach to fieldwork and data analysis that permits deep and recurrent questioning. Integrating the results ${ }^{35}$ will enable multilayered interrogation of differences and similarities generating greater insights into what has been identified and also to probe deeply into why.

There are challenges in generalising the findings to other settings and providing sufficiently detailed evidence for decision-makers to enact policy changes and implement appropriate services. Maximising the generalisability of the study findings requires both quantitative data about health improvement associated with the intervention and its associated costs, and in-depth qualitative data about the operationalisation of the intervention. There are several models of social prescribing and it is,

“... being implemented across the UK with local variations according to level and source of funding, model of commissioning, the targeting and identification of service users, geographical coverage, referral sources and the breadth of 'prescribed' activities." (Dayson 2017, p. 91)

Although social prescribing has become a more "mainstream' intervention in the UK since $2012,{ }^{40}$ commissioners and practitioners require robust evidence to guide the implementation of best practice social prescribing.

Acknowledgements We wish to thank Professor Chris Drinkwater, Dr Guy Pilkington, Ways to Wellness, First Contact Clinical, Mental Health Concern and North of England Commissioning Service and the service users who took part in the qualitative study.

Contributors SM, LP conceived of the study. All authors participated in its design: JW led the design of WPs 1 and 2, supported by JMW and MSP; TMP collaborated with SM and led the design of WP 3; SM, LP, JMW and NO'B designed the EQ-5D-5L study. SM drafted the manuscript and all authors contributed to its refinement. All authors have read and approved the final version.

Funding This work is funded by the National Institute of Health Research, Public Health Research Programme, Community Groups and Health Promotion (grant no. 16/122/33). The research was informed by a NIHR School for Public Health Research (SPHR) funded project (project reference: SPHR-FUS-PES-WTW).

Disclaimer The views expressed are those of the author(s) and not necessarily those of the NHS, the NIHR or the Department of Health and Social Care.

Competing interests None declared.

Patient consent for publication Not required.

Ethics approval UK NHS Integrated Research Approval System Ethics, Durham University Research Ethics Committee and Newcastle University Faculty of Medical Sciences Research Ethics Committee.

Provenance and peer review Not commissioned; peer reviewed for ethical and funding approval prior to submission.

Open access This is an open access article distributed in accordance with the Creative Commons Attribution Non Commercial (CC BY-NC 4.0) license, which permits others to distribute, remix, adapt, build upon this work non-commercially, and license their derivative works on different terms, provided the original work is properly cited, appropriate credit is given, any changes made indicated, and the use is non-commercial. See: http://creativecommons.org/licenses/by-nc/4.0/.

\section{REFERENCES}

1. Polley M, Bertotti M, Kimberlee R, et al. A review of the evidence assessing impact of social prescribing on healthcare demand and cost implications. London: University of Westminster, 2017.

2. South J, Higgins TJ, Woodall J, et al. Can social prescribing provide the missing link? Prim Health Care Res Dev 2008;9:310-8.

3. Social Prescribing Network. 2016. Report of the annual social prescribing network conference. London: University of Westminster.

4. Mossabir R, Morris R, Kennedy A, et al. A scoping review to understand the effectiveness of linking schemes from healthcare providers to community resources to improve the health and well-being of people with long-term conditions. Health Soc Care Community 2015;23:467-84.

5. Brandling J, House W. Social prescribing in general practice: adding meaning to medicine. Br J Gen Pract 2009;59:454-6.

6. NHS England. NHs five year forward view. $2014 \mathrm{https} / / / \mathrm{www}$. england.nhs.uk/publication/nhs-five-year-forward-view/

7. Department of Health and Social Care. New Deal for General Practice Jeremy Hunt sets out the first steps in a new deal for GPs. 
2015 https://www.gov.uk/government/speeches/new-deal-forgeneral-practice (accessed 16 May 2017).

8. Mahase E. New health secretary pledges $£ 4.5$ million towards GP social prescribing. Pulse. 2018 http://www.pulsetoday.co.uk/clinical/ clinical-specialties/prescribing/new-health-secretary-pledges-45mtowards-gp-social-prescribing/20037122.article

9. Bickerdike L, Booth A, Wilson PM, et al. Social prescribing: less rhetoric and more reality. A systematic review of the evidence. BMJ Open 2017;7:e013384.

10. Kilgarriff-Foster $A, O^{\prime}$ Cathain $A$. Exploring the components and impact of social prescribing. J Public Ment Health 2015;14:127-34.

11. Pilkington K, Loef M, Polley M. Searching for real-world effectiveness of health care innovations: scoping study of social prescribing for diabetes. J Med Internet Res 2017;19:e20.

12. Grant C, Goodenough T, Harvey I, et al. A randomised controlled tria and economic evaluation of a referrals facilitator between primary care and the voluntary sector. BMJ 2000;320:419-23.

13. Mercer SW, Fitzpatrick B, Grant L, et al. The Glasgow 'Deep End' Links Worker Study Protocol: a quasi-experimental evaluation of a social prescribing intervention for patients with complex needs in areas of high socioeconomic deprivation. J Comorb 2017;7:1-10.

14. Mercer SW, Wyke S, Fitzpatrick B, et al. Evaluation of the Glasgow 'Deep End' Links Worker Programme. Glasgow: Glasgow University, 2017.

15. Moffatt S, Penn L, O'Brien N, et al. Ways to Wellness: feasibility study of the impact of a social prescribing intervention: school for public health research, public health practitioner evaluation scheme. 2017 http://sphr.nihr.ac.uk/wp-content/uploads/2017/07/SPHR-FUSPES-WTW-final-report.pdf\#view=Fit (accessed 12 Dec 2017)

16. Moffatt $\mathrm{S}$, Steer $\mathrm{M}$, et al. What is the impact of 'social prescribing'? Perspectives of adults with long-term health conditions. BMJ Open 2017;0:e015203.

17 Moffatt S, Wildman J, Steer M, et al. Can 'Social prescribing' help adults with long term conditions to age well? International Association of Geriatrics and Gerontology 21st World Congress, San Franscisco, 2017.July 2017

18. Hex N, Bartlett $C$, Wright $D$, et al. Estimating the current and future costs of type 1 and Type 2 diabetes in the UK, including direct health costs and indirect societal and productivity costs. Diabet Med 2012;29:855-62.

19. NHS England. Business plan Leeds: NHS England. 2016 https:// www.england.nhs.uk/wp-content/uploads/2016/03/bus-plan-16.pdf.

20. Guthrie B, Payne K, Alderson P, et al. Adapting clinical guidelines to take account of multimorbidity. BMJ 2012;345:e6341.

21. Barnett K, Mercer SW, Norbury M, et al. Epidemiology of multimorbidity and implications for health care, research, and medical education: a cross-sectional study. Lancet 2012;380:37-43.

22. Department for Communities and Local Government. The English Indices of Deprivation. 2013.

23. Public Health England. Health Reports. 2015 http:// fingertipspheorguk/profile/health-profiles (accessed 16 Jan 2016).

24. Diabetes UK. Diabetes prevalence. Facts and Figures. 2016 https:// www.diabetes.org.uk/Professionals/Position-statements-reports/ Statistics/Diabetes-prevalence-2016 (accessed 20 Mar 2017).

25. NICE National Institute for Health and Care Excellence. Type 2 diabetes in adults: management. 2015 https://www.nice.org.uk/ guidance/ng28 (accessed 30 April 2017).

26. Morgan SL, Winship C. Counterfactuals and causal inference: methods and principles for social research. 2nd edn. Cambridge: Cambridge University Press, 2015.

27. Drummond MF, Sculpher MJ, Claxton K, et al; Methods for the economic evaluation of health care programmes. 4th edn. London: Oxford University Press, 2015.
28. NICE National Institute for Health and Care Excellence. Public health guidance: Smoking Cessation Services (PH10). Public health guidance. 2007 https://www.nice.org.uk/guidance/ph10

29. McEwan P, Evans M, Bergenheim K. A population model evaluating the costs and benefits associated with different oral treatment strategies in people with type 2 diabetes. Diabetes, Obesity and Metabolism 2010;12:623-30.

30. Drummond M, O'Brien B, Stoddart G, et al. Methods for the economic evaluation of health care programmes. 3rd edn: Oxford University Press, 2005

31. Oliffe JL, Bottorff JL. Further than the eye can see? Photo elicitation and research with men. Qual Health Res 2007;17:850-8.

32. Guell C, Ogilvie D. Picturing commuting: photovoice and seeking well-being in everyday travel. Qual Res 2015;15:201-18.

33. Thorne S. Data analysis in qualitative research. Evid Based Nurs 2000;3:68-70.

34. Pink S. Doing visual ethnography. London: Sage, 2013.

35. O'Cathain A, Murphy E, Nicholl J. Three techniques for integrating data in mixed methods studies. BMJ 2010;341:c4587.

36. Shadish WR, Cook TD, Campbell DT. Experimental and quasiexperimental designs for generalised causal inference. 2nd ed. Belmont, USA: Wadworth Publishing, 2001.

37. Craig P, Katikireddi SV, Leyland A, et al. Natural experiments: an overview of methods, approaches, and contributions to public health intervention research. Annu Rev Public Health 2017;38:39-56.

38. Rachel T, Robert B, Janet $\mathrm{H}$, et al. Critical moments: choice, chance and opportunity in young people's narratives of transition. Sociology 2002;36:335-54.

39. Kesten JM, Guell C, Cohn S, et al. From the concrete to the intangible: understanding the diverse experiences and impacts of new transport infrastructure. Int J Behav Nutr Phys Act 2015;12:72.

40. Dayson C. Social prescribing 'plus': a model of asset-based collaborative innovation? People, Place and Policy 2017;11:90-104.

41. ERS Research and Consultancy. Newcastle Social Prescribing Project. Final Report. 2013 http://www.healthworksnewcastle. org.uk/wp-content/plugins/downloads-manager/upload/Social\% 20Prescribing\%20Evaluation\%20Report\%20August $\% 202013 \%$ 20Final.pdf (accessed 16 Oct 2015).

42. Craig P, Dieppe P, Macintyre S, et al. Medical Research Council Guidance. Developing and evaluating complex interventions: the new Medical Research Council guidance. BMJ 2008;337:a1655.

43. NHS Year of Care Programme Team. Report of findings from the pilot programme. $2011 \mathrm{https}$ //www.yearofcare.co.uk/sites/default/ files/images/YOC Report\%20-\%20correct.pdf (accessed 14 Aug 2016).

44. Griffiths C, Foster G, Ramsay J, et al. How effective are expert patient (lay led) education programmes for chronic disease? BMJ 2007;334:1254-6.

45. Kennedy A, Reeves D, Bower P et al. The effectiveness and cost effectiveness of a national lay-led self care support programme for patients with long-term conditions: a pragmatic randomised controlled trial. J Epidemiol Community Health 2007;61:254-61.

46. Triangle Consulting Social Enterprise. The validity of the outcomes star as a tool for promoting and measuring service user change. 2014 http://static1.1.sqspcdn.com/static/f/312242/26519130/ $1441724321350 /$ Validity-and-reliability-of-the-Outcomes-StarSeptember+2015.pdf?token=J3I1FchTt9gHet2YfitaAq3LqL4\%3D (accessed 15 Nov 2016).

47. Rollnick S, Millar WR. Motivational interviewing in health care: helping patients change behavior (applications of motivational interviewing). London: Guildford Press, 2008. 